\title{
Development of nomograms to predict axillary lymph node status in breast cancer patients
}

\author{
Kai Chen ${ }^{1,2^{*+}} \mathbb{D}$, Jieqiong Liu ${ }^{1,2+}$, Shunrong $\mathrm{Li}^{1,2+}$ and Lisa Jacobs ${ }^{3^{*}}$
}

\begin{abstract}
Background: Prediction of axillary lymph node (ALN) status preoperatively is critical in the management of breast cancer patients. This study aims to develop a new set of nomograms to accurately predict ALN status.

Methods: We searched the National Cancer Database to identify eligible female breast cancer patients with profiles containing critical information. Patients diagnosed in 2010-2011 and 2012-2013 were designated the training ( $n=99,618)$ and validation $(n=101,834)$ cohorts, respectively. We used binary logistic regression to investigate risk factors for ALN status and to develop a new set of nomograms to determine the probability of having any positive ALNs and N2-3 disease. We used ROC analysis and calibration plots to assess the discriminative ability and accuracy of the nomograms, respectively.
\end{abstract}

Results: In the training cohort, we identified age, quadrant of the tumor, tumor size, histology, ER, PR, HER2, tumor grade and lymphovascular invasion as significant predictors of ALNs status. Nomogram-A was developed to predict the probability of having any positive ALNs (P_any) in the full population with a C-index of 0.788 and 0.786 in the training and validation cohorts, respectively. In patients with positive ALNs, Nomogram-B was developed to predict the conditional probability of having N2-3 disease (P_con) with a C-index of 0.680 and 0.677 in the training and validation cohorts, respectively. The absolute probability of having N2-3 disease can be estimated by P_any*P_con. Both of the nomograms were well-calibrated.

Conclusions: We developed a set of nomograms to predict the ALN status in breast cancer patients.

Keywords: Breast cancer, Nomogram, Lymph node status

\section{Background}

Treatment for early-stage breast cancer is focused on minimizing axillary surgery. The IBCSG 23-01 trial [1] demonstrated that patients with micrometastases in sentinel lymph nodes (SLNs) can be spared from axillary lymph node dissection (ALND). Furthermore, ALND does not provide any additional benefit in patients who received breast-conserving surgery (BCS) with 1-2 positive SLNs, as demonstrated in the Z11 trial [2]. Ongoing studies [3-5]

\footnotetext{
*Correspondence: chenkai23@mail.sysu.edu.cn; ljacob14@jhmi.edu

${ }^{\dagger}$ Equal contributors

'Guangdong Provincial Key Laboratory of Malignant Tumor Epigenetics and Gene Regulation, Sun Yat-Sen Memorial Hospital, Sun Yat-Sen University, Guangzhou, China

${ }^{3}$ Departments of Surgery and Oncology, Johns Hopkins Medical Institutions, Blalock \#607, 600 N. Wolfe St, Baltimore, Maryland 21287, USA

Full list of author information is available at the end of the article
}

are attempting to extend the results reported in the Z11 trial to mastectomy patients. The SOUND trial and the recent NCT01821768 trial [6] have been designed to explore the possibility of abandoning SLNB in a select group of patients [7]. However, the safety of the selection criteria used in these studies is unconfirmed. Predictive models for axillary lymph node (ALN) status would help to identify patients who are more likely to have negative ALNs to spare SLNB. These models, presented as nomograms, were reported and validated in different populations [8-11]. However, none has been widely accepted in clinical practice, possibly due to the lack of external validation in a large population.

In addition, most of the reported models were designed to predict the probability of having any positive ALNs $(\geq 1$ positive ALNs). It is also important to predict the probability 
of having N2-3 disease ( $>/=4$ positive ALNs) for clinical decision making. For example, in patients who fit the Z11 criteria and did not receive ALND, successful prediction of the axillary tumor burden may be informative for radiation oncologists in the determination of radiation fields.

The National Cancer Database (NCDB) is a joint program of the American College of Surgeons and the American Cancer Society. The database includes more than 1500 cancer programs in the United States with detailed tumor pathology information and overall survival data. Since 2010, data concerning HER2 status and lymphovascular invasion (LVI) have been available in the NCDB. In this study, we used data from the NCDB to develop novel and accurate nomograms that can predict the probability of having any positive ALNs and N2-3 disease. The wide range of patients represented in the NCDB may help to improve the robustness and generalizability of the novel nomograms.

\section{Methods}

\section{Patient selection}

We searched the NCDB registry dataset between 2010 and 2013 and identified female breast cancer patients using the following criteria:

\section{Inclusion criteria}

1) Year of diagnosis $\geq 2010$ (LVI and HER2 status have been available since 2010)

2) Female gender

3) A known number of lymph nodes was examined, and a known number of positive ALNs was reported

4) The location of the tumor was known (PRIMARY_SITE coding: C501;C502;C503; C504;C505)

\section{Exclusion criteria}

1) T-stage unknown, DCIS or T4 patients, or tumor size larger than $10 \mathrm{~cm}$.

2) Phyllodes tumor

3) Presence of metastatic disease at the time of diagnosis

4) Neoadjuvant chemotherapy

5) Patients with a prior tumor diagnosis

6) Patients with radical mastectomy, extended radical mastectomy or unknown surgery type

7) Bilateral breast cancer

8) Patients with overlapping lesions of the breast, multicentric lesions, or lesions that involved the entire breast (PRIMARY_SITE coding: C508;C509)

9) Tumor grade unknown, except for lobular carcinoma

10)ER, PR, and HER2 status unknown; HER2 borderline patients were also excluded

11)Unknown LVI status.
This was a retrospective study using anonymous and de-identified data from the NCDB. The authors cannot assess the information that could identify individual participants; therefore, this study was exempt from the Johns Hopkins Medicine Institutional Review Board and the Sun Yat-sen Memorial Hospital ethical committee review, and no consent was required.

\section{Statistical analysis}

Patients diagnosed from 2010 to 2011 and from 2012 to 2013 with $\geq 1$ nodes examined were defined as the training cohort and validation cohort, respectively, for predictive model development and validation.

We used the Chi-square test to identify risk factors for positive ALNs. The statistically significant $(P<0.001)$ risk factors were considered to be potential predictors of ALNs status and were all included in the full model. We used a binary logistic regression model to develop a predictive model for ALN status. We used Akaike information criterion (AIC) and ROC analysis to identify the optimal model. We used the full population to develop a prediction model (Model-A) of the risk of having any $\operatorname{ALNs}(+)$. Next, we developed a model (Model-B) that could estimate the conditional probability of having $\mathrm{pN} 2-3$, given the conditions that the patients had $\operatorname{ALNs}(+)$, that patients were ALN-positive, and that patients with $<10$ ALNs examined and $>=1$ positive ALNs $(N=23,106)$ were excluded.

We used the "rms" package of the R software to develop nomograms to visualize our predictive model graphically. Nomogram-A estimated the probability of having any positive ALNs (P_any). Nomogram-B estimated the conditional probability of having $\mathrm{pN} 2-3$ disease ( $\mathrm{P}_{-}$con). The probability of having $\mathrm{pN} 2-3$ disease can be calculated as P_any*P_con.

We used the ROC analysis and calibration plots to evaluate the discriminative ability and accuracy of the models, respectively. The performance of the models were evaluated and validated internally in the training cohort and externally in the validation cohort, respectively.

For sensitivity analysis, we randomly selected 500, 5000 and 50,000 patients from the study population and calculated the AUC values of the model in these subpopulations. We repeated the sampling for $N=200$ times and calculated the mean and standard deviations of the AUC values to determine the stability of AUC values.

All of the statistical analyses were performed using STATA 13.0MP and R.

\section{Results}

\section{Clinicopathological features}

This study included 201,452 breast cancer patients cataloged in the NCDB with a median age of 61 years old. The clinicopathological features are listed in Table 1. There were 99,618 and 101,834 patients in the training 
Table 1 Clincopathological features of the study populations

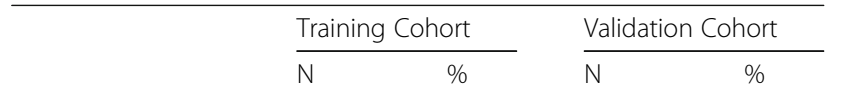

Year Of Diagnosis

$\begin{array}{lllll}2010 & 47,203 & 47.38 & 0 & 0.00 \\ 2011 & 52,415 & 52.62 & 0 & 0.00 \\ 2012 & 0 & 0.00 & 50,965 & 50.05 \\ 2013 & 0 & 0.00 & 50,869 & 49.95\end{array}$

Age Group

Age $<=50$ Yrs

$50-60 Y r s$

$>60 Y$ rs

23,231

23.32

22,203

21.80

50,420

26.07

26,600

26.12

52.08

Location Of Lesions

UIQ

UOQ

LOQ

LIQ

Central

Race

White
African American
Others

Unknown

T-Stage

$\mathrm{T}^{\mathrm{a}}$
$\mathrm{T} 2$
$\mathrm{~T} 3$

$\mathrm{N}$-Stage

No
N2
N3

Histology

IDC

ILC

IDC \& ILC

IDC \& Others

IMC

Others

Grade

।

III

Others/NA

Estrogen Receptor

Negative

$\begin{array}{llll}18,891 & 18.96 & 19,939 & 19.58 \\ 53,372 & 53.58 & 54,995 & 54.00 \\ 11,425 & 11.47 & 11,776 & 11.56 \\ 9086 & 9.12 & 8776 & 8.62 \\ 6844 & 6.87 & 6348 & 6.23\end{array}$

84,246

84.57

85,967

10,429

4568

870

854

0.86

69,375

27,675

69.64

27.78

71,740

27,528

2566

73,662

19,724

4313

1919

75,974

8582

5005

3359

1849

4849

25,663

43,908

29,420

627

15,746

15.81
Table 1 Clincopathological features of the study populations (Continued)

\begin{tabular}{|c|c|c|c|c|}
\hline Positive & 83,872 & 84.19 & 86,932 & 85.37 \\
\hline \multicolumn{5}{|c|}{ Progesterone Receptor } \\
\hline Negative & 25,080 & 25.18 & 23,426 & 23.00 \\
\hline Positive & 74,538 & 74.82 & 78,408 & 77.00 \\
\hline \multicolumn{5}{|l|}{ Her2 } \\
\hline Negative & 87,670 & 88.01 & 92,043 & 90.39 \\
\hline Positive & 11,948 & 11.99 & 9791 & 9.61 \\
\hline \multicolumn{5}{|c|}{ Lymphovascular Invasion } \\
\hline Not Present & 80,657 & 80.97 & 83,226 & 81.73 \\
\hline Present & 18,961 & 19.03 & 18,608 & 18.27 \\
\hline \multicolumn{5}{|c|}{ Charlson-Deyo Score } \\
\hline 0 & 83,641 & 83.96 & 84,466 & 82.94 \\
\hline 1 & 13,297 & 13.35 & 14,319 & 14.06 \\
\hline 2 & 2680 & 2.69 & 3049 & 2.99 \\
\hline \multicolumn{5}{|l|}{ Breast Surgery } \\
\hline $\mathrm{BCS}+\mathrm{RT}$ & 64,552 & 64.80 & 66,480 & 65.28 \\
\hline Mastectomy ${ }^{\mathrm{b}}$ & 35,066 & 35.20 & 35,354 & 34.72 \\
\hline \multicolumn{5}{|c|}{$\begin{array}{l}\text { NCDB national cancer database, Yrs years, HER2 human epidermal growth } \\
\text { factor receptor } 2, B C S \text { breast-conserving surgery, } R T \text { radiotherapy, LIQ lower- } \\
\text { inner quadrant, } L Q Q \text { lower-outer quadrant, UIQ Upper-inner quadrant, UOQ } \\
\text { Upper-outer quadrant, NA not available, IDC infiltrating ductal carcinoma, ILC } \\
\text { infiltrating lobular carcinoma, IMC invasive mucinous carcinoma; } \\
\text { aDCIS with micrometastasis (T1mic) were included in T1 } \\
\text { bS }\end{array}$} \\
\hline
\end{tabular}

and the validation cohort, respectively. Patient features were similar between the training cohort and the standard validation cohort.

\section{Nomogram for predicting risk of any positive ALNs}

We used Chi-square analysis and logistic regression as univariate and multivariate analysis to evaluate the risk factors for any positive ALNs in the training cohort. Age, location of lesions, T-stage, histology, ER, PR, HER2, tumor grade and LVI were independent predictors for any positive ALNs by univariate analysis (Table 2). These variables were further confirmed as independent factors in the multivariate analysis, and variables were incorporated in the full model. We also tested some variant models with different variables included. The full model had similar AIC and C-index with the variant model 2 (Additional file 1: Table S1) and the latter consisted of fewer variables. Therefore, we selected variant model 2 (with age, quadrant, size, histology, grade and LVI as predictors) for development of nomogram A to predict the risk of any positive ALNs (Fig. 1).

Nomogram for predicting pN2-3 disease in patients with any positive ALNs

We excluded patients with negative ALNs to predict the pN2-3 disease in patients with any positive ALNs. Patients had $<10$ ALNs nodes examined, and $\geq 1$ positive ALNs 
Table 2 Analysis of risk factors for any positive ALNs

\begin{tabular}{|c|c|c|c|c|c|c|c|}
\hline & \multicolumn{5}{|c|}{ Univariate analysis } & \multicolumn{2}{|c|}{ Multivariate analysis } \\
\hline & \multicolumn{2}{|c|}{ NO } & \multicolumn{2}{|l|}{$\mathrm{N} 1-3$} & \multirow[t]{2}{*}{$p^{b}$} & \multirow[t]{2}{*}{$\mathrm{OR}(95 \%)$} & \multirow[t]{2}{*}{$P$} \\
\hline & $\mathrm{n}$ & $\%^{a}$ & $\mathrm{n}$ & $\%^{a}$ & & & \\
\hline \multicolumn{8}{|l|}{ Age Group } \\
\hline Age $<=50$ Yrs & 15,652 & 67.38 & 7579 & 32.62 & $<0.001$ & 1 & \\
\hline $50-60 Y r s$ & 18,757 & 72.23 & 7210 & 27.77 & & $0.91(0.87-0.95)$ & $<0.001$ \\
\hline$>60 Y$ rs & 39,253 & 77.85 & 11,167 & 22.15 & & $0.70(0.67-0.73)$ & $<0.001$ \\
\hline \multicolumn{8}{|c|}{ Location Of Lesions } \\
\hline UIQ & 15,519 & 82.15 & 3372 & 17.85 & $<0.001$ & 1 & \\
\hline UOQ & 38,406 & 71.96 & 14,966 & 28.04 & & $1.84(1.75-1.93)$ & $<0.001$ \\
\hline LOQ & 8149 & 71.33 & 3276 & 28.67 & & $1.91(1.79-2.03)$ & $<0.001$ \\
\hline LIQ & 7089 & 78.02 & 1997 & 21.98 & & $1.46(1.36-1.56)$ & $<0.001$ \\
\hline Central & 4499 & 65.74 & 2345 & 34.26 & & $2.19(2.03-2.35)$ & $<0.001$ \\
\hline \multicolumn{8}{|l|}{ T-Stage } \\
\hline $\mathrm{T} 1$ & 57,744 & 83.23 & 11,631 & 16.77 & $<0.001$ & 1 & \\
\hline $\mathrm{T} 2$ & 15,160 & 54.78 & 12,515 & 45.22 & & $3.05(2.95-3.16)$ & $<0.001$ \\
\hline $\mathrm{T} 3$ & 758 & 29.52 & 1810 & 70.48 & & $7.85(7.12-8.65)$ & $<0.001$ \\
\hline \multicolumn{8}{|l|}{ Histology } \\
\hline IDC & 55,824 & 73.48 & 20,150 & 26.52 & $<0.001$ & 1 & \\
\hline ILC & 6174 & 71.94 & 2408 & 28.06 & & $1.08(1.02-1.15)$ & 0.009 \\
\hline IDC \& ILC & 3437 & 68.67 & 1568 & 31.33 & & $1.19(1.11-1.28)$ & $<0.001$ \\
\hline IDC \& Others & 2688 & 80.02 & 671 & 19.98 & & $0.74(0.67-0.82)$ & $<0.001$ \\
\hline IMC & 1731 & 93.62 & 118 & 6.38 & & $0.24(0.19-0.29)$ & $<0.001$ \\
\hline Others & 3808 & 78.53 & 1041 & 21.47 & & $0.79(0.73-0.86)$ & $<0.001$ \\
\hline \multicolumn{8}{|l|}{ Grade } \\
\hline 1 & 21,746 & 84.74 & 3917 & 15.26 & $<0.001$ & 1 & \\
\hline$\|$ & 32,129 & 73.17 & 11,779 & 26.83 & & $1.29(1.23-1.35)$ & $<0.001$ \\
\hline III & 19,337 & 65.73 & 10,083 & 34.27 & & $1.31(1.24-1.38)$ & $<0.001$ \\
\hline Others/NA & 450 & 71.77 & 177 & 28.23 & & $1.27(1.04-1.56)$ & 0.022 \\
\hline \multicolumn{8}{|l|}{ Estrogen Receptor } \\
\hline Negative & 11,326 & 71.93 & 4420 & 28.07 & $<0.001$ & 1 & \\
\hline Positive & 62,336 & 74.32 & 21,536 & 25.68 & & $1.20(1.12-1.28)$ & $<0.001$ \\
\hline \multicolumn{8}{|c|}{ Progesterone Receptor } \\
\hline Negative & 18,233 & 72.70 & 6847 & 27.30 & $<0.001$ & 1 & \\
\hline Positive & 55,429 & 74.36 & 19,109 & 25.64 & & $1.16(1.10-1.23)$ & $<0.001$ \\
\hline \multicolumn{8}{|l|}{ Her2 } \\
\hline Negative & 65,656 & 74.89 & 22,014 & 25.11 & $<0.001$ & 1 & \\
\hline Positive & 8006 & 67.01 & 3942 & 32.99 & & $1.11(1.05-1.16)$ & $<0.001$ \\
\hline \multicolumn{8}{|c|}{ Lymphovascular Invasion } \\
\hline Not Present & 66,799 & 82.82 & 13,858 & 17.18 & $<0.001$ & 1 & \\
\hline Present & 6863 & 36.20 & 12,098 & 63.80 & & $6.36(6.12-6.60)$ & $<0.001$ \\
\hline
\end{tabular}

ALN axillary lymph node, Yrs years old, HER2 human epidermal growth factor receptor 2, LIQ lower-inner quadrant, LOQ lower-outer quadrant, UIQ Upper-inner quadrant, UOQ Upper-outer quadrant, N/A not available, IDC infiltrating ductal carcinoma, ILC infiltrating lobular carcinoma, IMC invasive mucinous carcinoma, NS non-significant

${ }^{a}$ Row percentage was shown

${ }^{\mathrm{b}} \mathrm{Chi}$-square test was used for univariate analysis 


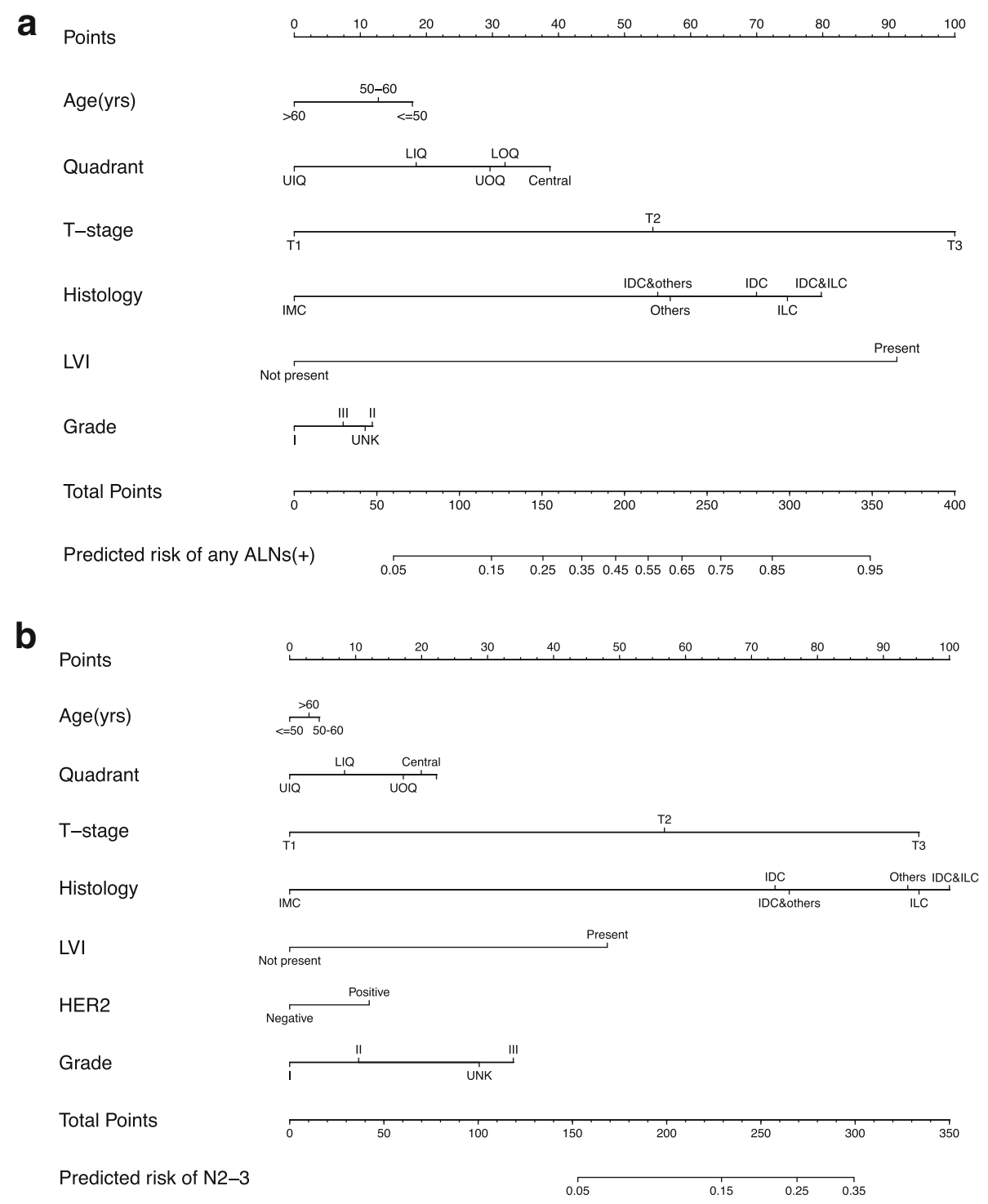

Fig. 1 a Nomogram to predict the probability of having any positive ALNs (P_any); b Nomogram to predict the conditional probability of having N2-3 disease (P_con), when the patients have any positive ALNs. The absolute probability of having N2-3 can be estimated by P_any*P_con

were also excluded $(N=23,106)$. Univariate analysis suggested that age, location of lesions, T-stage, histology, ER, PR, HER2, tumor grade and LVI were risk factors for pN23 disease in patients with any positive ALNs (Table 3). These variables, except for ER and PR status, were confirmed as independent risk factors in the multivariate analysis. The full model was selected based on its lowest AIC and the highest C-index (Additional file 1: Table S1). Nomogram-B (Fig. 1) was developed to predict the conditional probability of having $\mathrm{pN} 2-3$ patients, given that patients have $\geq 1$ positive ALNs.

\section{Distribution of the predicted probability}

The training cohort and the validation cohort exhibited a similar distribution of predicted risks by the new model (Additional file 2: Fig. S1). Most of the predicted risk of any ALNs ranged between 0 and $20 \%$. Most of the predicted risks of $\mathrm{pN} 2-3$ disease ranged between $10 \%$ and $50 \%$ and between $0 \%$ and $10 \%$ in patients with any positive ALNs and in all populations, respectively.

\section{Validation of the nomograms}

The AUC values of the nomograms (Additional file 1: Table S1) for predicting any positive ALNs and pN2-3 disease were 0.788 and 0.680 in the training cohort and 0.786 and 0.677 in the validation cohort, respectively. The calibration plot (Fig. 2) suggested that the nomograms were well-calibrated. The average estimation errors of predicting any positive ALNs and pN2-3 
Table 3 Analysis of risk factors for pN2-3 $3^{a}$

\begin{tabular}{|c|c|c|c|c|c|c|c|}
\hline & \multicolumn{5}{|c|}{ Univariate analysis } & \multicolumn{2}{|c|}{ Multivariate analysis } \\
\hline & \multicolumn{2}{|c|}{ NO } & \multicolumn{2}{|l|}{ N1-3 } & \multirow[t]{2}{*}{$p^{b}$} & \multirow[t]{2}{*}{$\mathrm{OR}(95 \%)$} & \multirow[t]{2}{*}{$P$} \\
\hline & $\bar{n}$ & $\%^{\mathrm{a}}$ & $\mathrm{n}$ & $\%+$ & & & \\
\hline \multicolumn{8}{|l|}{ Age Group } \\
\hline Age $<=50$ Yrs & 3229 & 66.30 & 1641 & 33.70 & $<0.001$ & 1 & \\
\hline $50-60 Y r s$ & 2958 & 66.20 & 1510 & 33.80 & & $1.06(0.97-1.16)$ & 0.202 \\
\hline$>60 Y r s$ & 3917 & 62.49 & 2351 & 37.51 & & $1.23(1.14-1.34)$ & $<0.001$ \\
\hline \multicolumn{8}{|c|}{ Location Of Lesions } \\
\hline UIQ & 1374 & 69.39 & 606 & 30.61 & $<0.001$ & 1 & \\
\hline UOQ & 5753 & 64.16 & 3213 & 35.84 & & $1.30(1.17-1.45)$ & $<0.001$ \\
\hline LOQ & 1300 & 63.82 & 737 & 36.18 & & $1.38(1.20-1.59)$ & $<0.001$ \\
\hline LIQ & 788 & 69.31 & 349 & 30.69 & & $1.15(0.98-1.36)$ & 0.095 \\
\hline Central & 889 & 59.83 & 597 & 40.17 & & $1.36(1.17-1.58)$ & $<0.001$ \\
\hline \multicolumn{8}{|l|}{ T-Stage } \\
\hline $\mathrm{T} 1$ & 4589 & 76.36 & 1421 & 23.64 & $<0.001$ & 1 & \\
\hline $\mathrm{T} 2$ & 4993 & 60.53 & 3256 & 39.47 & & $1.83(1.69-1.97)$ & $<0.001$ \\
\hline T3 & 522 & 38.75 & 825 & 61.25 & & $3.97(3.48-4.52)$ & $<0.001$ \\
\hline \multicolumn{8}{|l|}{ Histology } \\
\hline IDC & 8027 & 66.32 & 4076 & 33.68 & $<0.001$ & 1 & \\
\hline ILC & 761 & 52.02 & 702 & 47.98 & & $1.96(1.73-2.22)$ & $<0.001$ \\
\hline IDC \& ILC & 570 & 61.16 & 362 & 38.84 & & $1.27(1.10-1.47)$ & 0.001 \\
\hline IDC \& Others & 272 & 67.83 & 129 & 32.17 & & $0.91(0.73-1.13)$ & 0.396 \\
\hline $\mathrm{IMC}$ & 59 & 81.94 & 13 & 18.06 & & $0.44(0.24-0.82)$ & 0.01 \\
\hline Others & 415 & 65.35 & 220 & 34.65 & & $1.02(0.85-1.21)$ & 0.848 \\
\hline \multicolumn{8}{|l|}{ Grade } \\
\hline । & 1482 & 75.88 & 471 & 24.12 & $<0.001$ & 1 & \\
\hline$\|$ & 4433 & 65.51 & 2334 & 34.49 & & $1.30(1.15-1.47)$ & $<0.001$ \\
\hline III & 4140 & 60.98 & 2649 & 39.02 & & $1.44(1.26-1.64)$ & $<0.001$ \\
\hline Others/NA & 49 & 50.52 & 48 & 49.48 & & $1.48(0.95-2.30)$ & 0.08 \\
\hline \multicolumn{8}{|c|}{ Estrogen Receptor } \\
\hline Negative & 1892 & 62.18 & 1151 & 37.82 & $<0.001$ & 1 & \\
\hline Positive & 8212 & 65.37 & 4351 & 34.63 & & $1.05(0.92-1.19)$ & 0.467 \\
\hline \multicolumn{8}{|c|}{ Progesterone Receptor } \\
\hline Negative & 2809 & 61.93 & 1727 & 38.07 & $<0.001$ & 1 & \\
\hline Positive & 7295 & 65.90 & 3775 & 34.10 & & $0.93(0.84-1.04)$ & 0.219 \\
\hline \multicolumn{8}{|l|}{ Her2 } \\
\hline Negative & 8541 & 65.96 & 4408 & 34.04 & $<0.001$ & 1 & \\
\hline Positive & 1563 & 58.83 & 1094 & 41.17 & & $1.30(1.18-1.42)$ & $<0.001$ \\
\hline \multicolumn{8}{|c|}{ Lymphovascular Invasion } \\
\hline Not Present & 5632 & 74.12 & 1966 & 25.88 & $<0.001$ & 1 & \\
\hline Present & 4472 & 55.84 & 3536 & 44.16 & & $2.11(1.96-2.26)$ & $<0.001$ \\
\hline
\end{tabular}

ALN axillary lymph node, Yrs years old, HER2 human epidermal growth factor receptor 2, LIQ lower-inner quadrant, LOQ lower-outer quadrant, UIQ Upper-inner quadrant, UOQ Upper-outer quadrant, N/A not available, IDC infiltrating ductal carcinoma, ILC infiltrating lobular carcinoma, IMC invasive mucinous carcinoma, NS non-significant

${ }^{a}$ Only patients with positive nodes were included. Patients with $<10$ axillary lymph nodes examined $<10$ but $>1$ positive ALNs were excluded

${ }^{\mathrm{b}}$ Row percentage was shown.

${ }^{* * *}$ Chi-square test was used for univariate analysis 

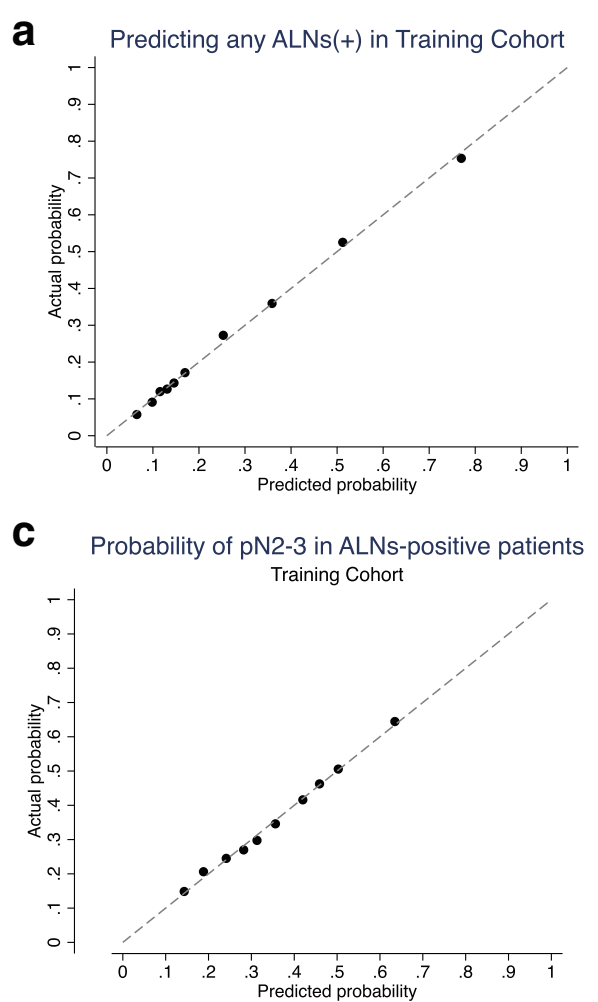

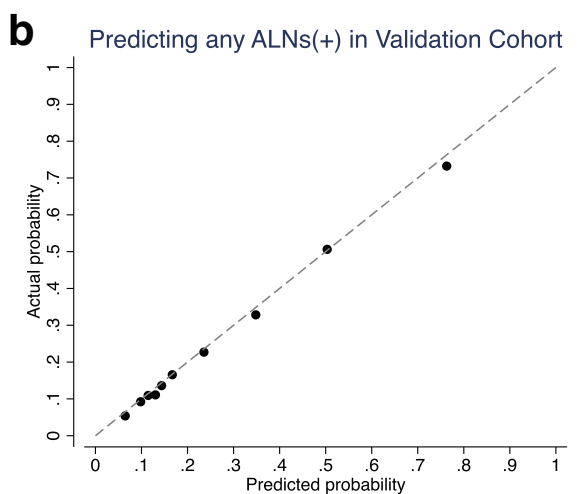

d
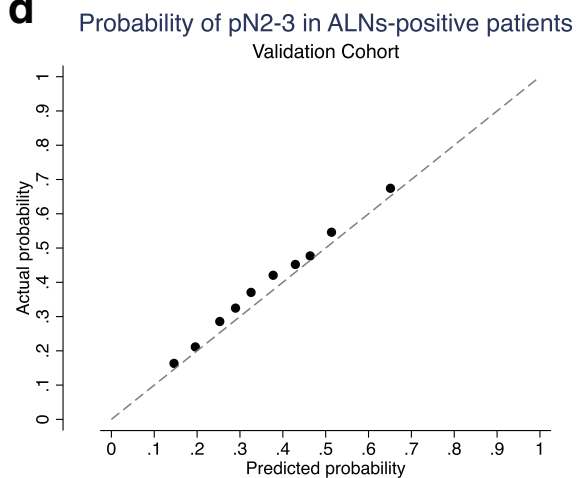

Fig. 2 Calibration plots of nomogram-A to predict the probability of having any positive ALNs in the a) training and $\mathbf{b}$ ) validation cohort, and nomogram-B to predict the conditional probability of having N2-3 disease in the c) training and $\mathbf{d}$ ) validation cohort

disease were $0.78 \%$ and $0.85 \%$ in the training cohort and $1.14 \%$ and $2.79 \%$ in the validation cohort, respectively.

\section{Sensitivity analysis}

For sensitivity analysis, we randomly selected 500, 5000 and 50,000 patients from the training and validation cohorts and performed the ROC analysis and calibration plot analysis. We repeated the re-sampling 200 times to obtain a reliable estimation of the AUC values and average prediction error between the actual and predicted risks. As shown in Additional file 3: Table S2, the estimated AUC values and average prediction error were similar among sub-populations with varied sample sizes.

\section{Discussion}

\section{Accuracy of the nomograms}

The first predictive model for ALN status was developed a decade ago by Bevilacqua et al. [8]. The authors retrospectively reviewed the database of MSKCC and identified 3786 and 1545 breast cancer patients as training and validation sets, respectively. A nomogram was developed using age, tumor size, special pathology type, location, LVI, multifocal status, nuclear grade, and ER and PR status as predictors of ALN status. Chen et al. [10] validated the MSKCC model in a Chinese population $(n=1545)$ and reported a new nomogram (the Shanghai model) using data from Chinese breast cancer patients. However, the MSKCC model did not incorporate HER2 status. Reyal et al. [11] reported that molecular subtype approximation, including ER, PR and HER2, is also a determinant of ALN status, and another nomogram was later developed (the Paris model). Additionally, several more models [9, 12-15] have been developed to predict ALN status. However, none of these models has been widely accepted by treatment guidelines, and clinical practice has not significantly changed. A lack of sufficient evidence to support external validity is one of the major underlying reasons. In addition, these models can only predict the risk of having $\geq 1$ positive ALN. In the current study, we used a large multi-institutional NCDB population to develop and validate a set of nomograms that can predict the risk of having any positive ALNs and N2-3 disease.

\section{Benefit of the new nomograms in the post-Z0011 era}

The Z11 study [2] demonstrated that patients with 1-2 positive SLNs receiving BCS and standardized adjuvant therapies could be spared from ALND [16]. However, it is impossible to know whether a patient fits the Z11 criteria or not before surgery, as the number of positive SLNs can only be identified during or after surgery. Our nomograms may be able to identify patients who may 
not fit the Z11 criteria by predicting the risk of having $\mathrm{N} 2-3$ disease preoperatively. If a patient had a high risk of having N2-3 disease, she may be unlikely to fit the Z11 criteria.

Because mastectomy patients were not included in the Z11 study, ALND is still a routine procedure for SLNpositive patients. However, several retrospective studies suggested the feasibility to omit ALND in selected mastectomy patients with positive $[4,17,18]$. A prospective randomized trial was also initiated to test this hypothesis (NCT02112682). Therefore, the trend that Z11 conclusions could be extended to mastectomy patients is very clear, and with the help of these nomograms, surgeons may feel safer in omitting the ALND in selected mastectomy patients with positive SLNs.

One concern related to omitting the ALND in mastectomy patients is whether RT should be given. The NCCN guidelines [19] clearly recommend RT to the infraclavicular region, supraclavicular area, and internal mammary nodes for patients with $\mathrm{N} 2-3$ disease ( $\geq 4$ positive ALNs). For patients with $1-3$ positive nodes (N1 disease) after mastectomy, radiotherapy coverage of these areas was considered controversial by NCCN panel members [19] because high-level contradictory evidence was apparent [20-23]. With our nomograms, omitting ALND in selected mastectomy patients after positive SLNs may not be a major problem, as the radiation oncologist can estimate the risk of having N2-3 disease and determine the treatment plans. Additionally, these nomograms would be more helpful to the radiation oncologists, in that 1) they may help reassure them that patients who met Z11 criteria do not need additional radiation therapy, in terms of increasing the tangents/fields for radiation; 2) For patients who received neoadjuvant chemotherapy, RT decisions should be based on pre-chemotherapy tumor features regardless of the tumor response [18]. Our nomograms may be useful in the estimation of axillary tumor burden prior to the initiation of neoadjuvant chemotherapy to provide more information.

Benefit of the new nomograms in the "SLNB-sparing" era In the post-Z11 era when "the days are numbered for axillary surgery" [24], it is likely that SLNB could be omitted in selected patients. The SOUND trial [7], proposed in 2012, was designed to test this hypothesis. In the SOUND trial, T1 breast cancer patients with clinically negative axilla were randomized into groups receiving either observation or SLNB. There were only $12.8 \%$ of patients with positive ALNs in the SLNB group, suggesting the high probability that SLNB could be spared in the future. The development of the nomograms is consistent with the trend towards the "SLNB-sparing" era. If we could identify node negative patients preoperatively, the omission of SLNB would be much safer, without the need to wait for the results of the SOUND trial. Sparing SLNB would improve the quality of life and reduce the medical cost and all possible surgical complications. The authors recently reported that the physical function of the upper limb in the no-SLNB group was significantly better than in the SLNB-group, suggesting the benefit of minimizing axillary surgery when appropriate [25].

In the "SLNB-sparing" era, if the SOUND trial demonstrated that not performing SLNB in selected patients is safe, there will be concerns regarding the absence of axillary staging on the decision to use adjuvant therapies. For example, post-mastectomy radiotherapy (PMRT) is not necessary in T1-2 patients with negative ALNs, whereas in patients with positive ALNs, PMRT is strongly recommended by the NCCN guidelines [19]. The need for radiotherapy has also influenced the optimal timing of breast reconstruction (e.g., immediate vs. delayed). Additionally, a T1a patient with HER2 positive disease may be spared from chemotherapy if ALNs were negative, and adjuvant chemotherapy is recommended for patients with positive ALNs [19]. Taken together, the ability to predict the probability of having any positive ALNs (or N0 disease) would be helpful in the "SLNBsparing" era in the future.

\section{Limitations}

There are several limitations to this study.

First, several of the predictors of these models, such as LVI and multifocal lesions, may not always be available prior to surgery. Core needle biopsy may not provide an adequate volume of tissue for the identification of LVI. These issues may limit the utility of the models developed in the current study. However, ultrasound-guided vacuum-assisted biopsy [26, 27] has been used by many institutions and may provide a larger volume of tissue for the identification of LVI. Current imaging modalities can provide an accurate estimate of tumor size and multifocality [28-31].

Second, several important variables are not available in the NCDB, such as whether the tumor was palpable or ki-67 status. More importantly, the clinical axillary status was also not available in this study. In patients with clinically negative axilla, the probability of having $\mathrm{N} 2-3$ disease is very low. The performance of the model in these patients needs to be validated.

Third, the NSABP B-32 trial suggested a $10 \%$ falsenegative rate of SLNs. In our study, patients with SLNB only, without any positive SLNs, were classified as having no positive ALNs. This limitation cannot be avoided when using data from the modern era when SLNB is the routine practice. However, we believe that the $10 \%$ false-negative rate may not significantly affect the performance of our model. 
Fourth, when developing nomogram-B for predicting the conditional probability of having $\mathrm{N} 2-3$ disease, we excluded 23,106 patients $(11.5 \%, 23,106 / 201,452)$ with $\geq 1$ positive ALNs but with less than 10 ALNs evaluated. We may have skewed the data by excluding these patients. However, we considered that the benefit of excluding these patients might outweigh the harm of including them. As demonstrated in the Z11 study, patients with 1$2 \mathrm{SLN}+$ without further ALND may theoretically have a $27 \%$ risk of additional positive ALNs. Therefore, the exact amount of positive ALNs in these patients was unknown, leading to the inaccuracy of model development and validation.

Fifth, these nomograms can only be used in patients with a single focus of disease and only in patients with unilateral disease.

\section{Conclusions}

In this study, we used a large multi-institutional NCDB population to develop a set of nomograms to predict nodal status in breast cancer patients. Future validation studies are needed to confirm our findings.

\section{Additional files}

Additional file 1: Table S1. Model performance of the full models and the variants. This table showed the performance of the full models and their variants. (XLSX $42 \mathrm{~kb}$ )

Additional file 2: Figure S1. Kernel density plots of the a) predicted probability of having any positive ALNs by nomogram-A, b) predicted conditional probability of having N2-3 disease in patients with positive ALNs by nomogram- $B$, and $c$ ) the predicted absolute probability of having N2-3 disease in all populations, by nomogram-A and B. (PDF $54 \mathrm{~kb}$ )

Additional file 3: Table S2. Sensitivity analysis of the model performance in population with different sample sizes. We used different populations with different sample size to assess the performance and the robustness of the models. (XLSX $41 \mathrm{~kb}$ )

\section{Abbreviations}

AIC: Akaike information criterion; ALN: Axillary lymph node; ALND: Axillary lymph node dissection; AUC: Area under the curve; BCS: Breast conserving surgery; ER: Estrogen receptor; HER2: Human epidermal growth receptor 2; LVI: Iymphovascular invasion; NCDB: National Cancer Database; PMRT: Postmastectomy radiotherapy; PR: Progesterone receptor; ROC: Receiveroperator-curve; SLN: Sentinel lymph node

\section{Acknowledgements}

The data used in the study was derived from a de-identified NCDB. The American College of Surgeons and the Commission on Cancer have not verified and are not responsible for the analytic or statistical methodology used or the conclusions drawn from these data by the investigator. This study was supported by the National Natural Science Foundation of China (Grant \# 81402201, Grant \# 81602673), and Grant [2013] 163 from the Key Laboratory of Malignant Tumor Molecular Mechanism and Translational Medicine of Guangzhou Bureau of Science and Information Technology.

\section{Funding}

This study was supported by the National Natural Science Foundation of China (Grant \# 81402201/81372817) and Grant [2013] 163 from Key Laboratory of Malignant Tumor Molecular Mechanism and Translational Medicine of Guangzhou Bureau of Science and Information Technology. All of the funding bodies did not have a role in the design of the study and data collection or analysis. Also they did not have a role in the interpretation of the data. The funding bodies supported the travel of the author (Kai Chen) to US to meet with Lisa Jacobs for discussion over this manuscript. Also, the funding bodies support the expense of the language editting of this manuscript, and the working allowance for the authors (Kai Chen, Jiegiong Liu and Shunrong Li).

\section{Availability of data and materials}

We used the NCDB data and selected appropriate patients for this study. We only retrieved relevant variables for the included patients, and we cleaned the data, stored it as the dta. File, which can be loaded using the STATA 13. As the author had signed an agreement with the NCDB and promise not to share the data with others, we cannot upload the "clean" data online. However, for scientific purpose, the "clean" data are freely available upon request. Anyone who wants to request the data can contact the corresponding author Kai Chen (chenkai23@mail.sysu.edu.cn) and need to sign a Data Transfer Agreement with Kai Chen, Lisa Jacobs, and possibly the NCDB.

Ethics approval and consent to participate.

This is a study using a publicly available database (NCDB) with signed agreement between the authors and the NCDB. All of the data are anonymous. Therefore, the ethical approval can be waived, based on our institutional policy and consent to participate and is not needed.

\section{Authors' contributions}

K.C. made substantial contribution to conception and design, analysis and interpretation of data, wrote and revised the manuscript, gave final approval for publications and agreed to be accountable for all aspects of the work. J.L. collected and analyzed the data, wrote and revised the manuscript, gave final approval for publications and agreed to be accountable for all aspects of the work. S.L. designed the study and analyzed the data, and also took part in the drafting of the manuscript, gave final approval for publications and agreed to be accountable for all aspects of the work. L.J. helped to collect the data, and get invovled in analyzing the data, gave critical and important opinions in the revision of the manusript, gave final approval for publications and agreed to be accountable for all aspects of the work.

Consent for publication

Not applicable.

\section{Competing interests}

The authors declare that they have no competing interests in this section.

\section{Publisher's Note}

Springer Nature remains neutral with regard to jurisdictional claims in published maps and institutional affiliations.

\section{Author details}

'Guangdong Provincial Key Laboratory of Malignant Tumor Epigenetics and Gene Regulation, Sun Yat-Sen Memorial Hospital, Sun Yat-Sen University, Guangzhou, China. ${ }^{2}$ Breast Tumor Center, Sun Yat-sen Memorial Hospital, Sun Yat-sen University, Guangzhou, Guangdong 510120, China. ${ }^{3}$ Departments of Surgery and Oncology, Johns Hopkins Medical Institutions, Blalock \#607, 600 N. Wolfe St, Baltimore, Maryland 21287, USA.

Received: 16 May 2017 Accepted: 3 August 2017

Published online: 23 August 2017

References

1. Galimberti V, Cole BF, Zurrida S, Viale G, Luini A, Veronesi P, Baratella P, Chifu C, Sargenti M, Intra M, et al. Axillary dissection versus no axillary dissection in patients with sentinel-node micrometastases (IBCSG 23-01): a phase 3 randomised controlled trial. Lancet Oncol. 2013;14(4):297-305.

2. Giuliano AE, Hunt KK, Ballman KV, Beitsch PD, Whitworth PW, Blumencranz PW, Leitch AM, Saha S, McCall LM, Morrow M. Axillary dissection vs no axillary dissection in women with invasive breast cancer and sentinel node metastasis: a randomized clinical trial. JAMA. 2011;305(6):569-75.

3. Crawford JD, Ansteth M, Barnett J, Glissmeyer M, Johnson NG. Routine completion axillary lymph node dissection for positive sentinel nodes in patients undergoing mastectomy is not associated with improved local control. Am J Surg. 2013;205(5):581-4. discussion 584 
4. Fu Y, Chung D, Cao MA, Apple S, Chang H. Is axillary lymph node dissection necessary after sentinel lymph node biopsy in patients with mastectomy and pathological N1 breast cancer? Ann Surg Oncol. 2014;21(13):4109-23.

5. Omitting Completion Axillary Treatment in Sentinel Node Positive Breast Cancer Patients Undergoing a Mastectomy. https://clinicaltrials.gov/ct2/ show/NCT02112682

6. Axillary Ultrasound With or Without Sentinel Lymph Node Biopsy in Detecting the Spread of Breast Cancer in Patients Receiving Breast Conservation Therapy. https://clinicaltrials.gov/ct2/show/NCT01821768

7. Gentilini $\mathrm{O}$, Veronesi U. Abandoning sentinel lymph node biopsy in early breast cancer? A new trial in progress at the European Institute of Oncology of Milan (SOUND: Sentinel node vs Observation after axillary UltraSouND). Breast. 2012;21(5):678-81.

8. Bevilacqua JL, Kattan MW, Fey JV, Cody HS 3rd, Borgen PI, Van Zee KJ. Doctor, what are my chances of having a positive sentinel node? A validated nomogram for risk estimation. J Clin Oncol. 2007;25(24):3670-9.

9. Carmichael AR, Aparanji K, Nightingale P, Boparai R, Stonelake PS. A clinicopathological scoring system to select breast cancer patients for sentinel node biopsy. Eur J Surg Oncol. 2006;32(10):1170-4.

10. Chen JY, Chen JJ, Yang BL, Liu ZB, Huang XY, Liu GY, Han QX, Yang WT, Shen ZZ, Shao ZM, et al. Predicting sentinel lymph node metastasis in a Chinese breast cancer population: assessment of an existing nomogram and a new predictive nomogram. Breast Cancer Res Treat. 2012;135(3):839-48.

11. Reyal F, Rouzier R, Depont-Hazelzet B, Bollet MA, Pierga JY, Alran S, Salmon RJ, Fourchotte V, Vincent-Salomon A, Sastre-Garau X, et al. The molecular subtype classification is a determinant of sentinel node positivity in early breast carcinoma. PLoS One. 2011;6(5):e20297.

12. Kolarik D, Pecha V, Skovajsova M, Zahumensky J, Trnkova M, Petruzelka L, Halaska M, Sottner O, Otcenasek M, Kolarova H. Predicting axillary sentinel node status in patients with primary breast cancer. Neoplasma. 2013;60(3):334-42.

13. Qiu SQ, Zeng HC, Zhang F, Chen C, Huang WH, Pleijhuis RG, Wu JD, van Dam GM, Zhang GJ. A nomogram to predict the probability of axillary lymph node metastasis in early breast cancer patients with positive axillary ultrasound. Sci Rep. 2016;6:21196.

14. Xie F, Yang H, Wang S, Zhou B, Tong F, Yang D, Zhang J. A logistic regression model for predicting axillary lymph node metastases in early breast carcinoma patients. Sensors. 2012;12(7):9936-50.

15. Fehm T, Maul H, Gebauer S, Scharf A, Baier P, Sohn C, Jager W, Gebauer G. Prediction of axillary lymph node status of breast cancer patients by tumorbiological factors of the primary tumor. Strahlenther Onkol. 2005; 181(9):580-6.

16. Wright GP, Mater ME, Sobel HL, Knoll GM, Oostendorp LD, Melnik MK, Chung $\mathrm{MH}$. Measuring the impact of the American College of Surgeons Oncology Group Z0011 trial on breast cancer surgery in a community health system. Am J Surg. 2015;209(2):240-5.

17. Milgrom S, Cody H, Tan L, Morrow M, Pesce C, Setton J, Rogers K, Arnold B, Eaton A, Catalano J, et al. Characteristics and outcomes of sentinel nodepositive breast cancer patients after total mastectomy without axillaryspecific treatment. Ann Surg Oncol. 2012;19(12):3762-70.

18. Cowher MS, Grobmyer SR, Lyons J, O'Rourke C, Baynes D, Crowe JP. Conservative axillary surgery in breast cancer patients undergoing mastectomy: long-term results. J Am Coll Surg. 2014;218(4):819-24.

19. NCCN Clinical Practice Guidelines in Oncology. Breast Cancer. Version I. 2017. www.ncen.org

20. Clarke M, Collins R, Darby S, Davies C, Elphinstone P, Evans V, Godwin J, Gray R, Hicks C, James $S$, et al. Effects of radiotherapy and of differences in the extent of surgery for early breast cancer on local recurrence and 15-year survival: an overview of the randomised trials. Lancet. 2005;366(9503):2087-106.

21. Ragaz J, Olivotto IA, Spinelli JJ, Phillips N, Jackson SM, Wilson KS, Knowling MA, Coppin CM, Weir L, Gelmon K, et al. Locoregional radiation therapy in patients with high-risk breast cancer receiving adjuvant chemotherapy: 20-year results of the British Columbia randomized trial. J Natl Cancer Inst. 2005;97(2):116-26.

22. Recht A, Edge SB, Solin $\sqcup$, Robinson DS, Estabrook A, Fine RE, Fleming GF, Formenti S, Hudis C, Kirshner JJ, et al. Postmastectomy radiotherapy: clinical practice guidelines of the American Society of Clinical Oncology. J Clin Oncol. 2001;19(5):1539-69.

23. Overgaard M, Jensen MB, Overgaard J, Hansen PS, Rose C, Andersson M, Kamby C, Kjaer M, Gadeberg CC, Rasmussen BB, et al. Postoperative radiotherapy in high-risk postmenopausal breast-cancer patients given adjuvant tamoxifen: Danish Breast Cancer Cooperative Group DBCG 82C randomised trial. Lancet. 1999:353(9165):1641-8.

24. Gentilini O, Veronesi U. Staging the Axilla in Early Breast Cancer: Will Imaging Replace Surgery? JAMA Oncol. 2015;1(8):1031-2.

25. Gentilini O, Botteri E, Dadda P, Sangalli C, Boccardo C, Peradze N, Ghisini R, Galimberti V, Veronesi P, Luini A, et al. Physical function of the upper limb after breast cancer surgery. Results from the SOUND (Sentinel node vs. Observation after axillary Ultra-souND) trial. Eur J Surg Oncol. 2016;42(5):685-9.

26. Meyer JE, Smith DN, Lester SC, Kaelin C, PJ DP, Denison CM, Christian RL, Harvey SC, Selland DL, Durfee SM. Large-core needle biopsy of nonpalpable breast lesions. JAMA. 1999;281(17):1638-41.

27. Preibsch H, Baur A, Wietek BM, Kramer B, Staebler A, Claussen CD, Siegmann-Luz KC. Vacuum-assisted breast biopsy with 7-gauge, 8-gauge, 9gauge, 10-gauge, and 11-gauge needles: how many specimens are necessary? Acta Radiol. 2015:56(9):1078-84.

28. Fiorentino C, Berruti A, Bottini A, Bodini M, Brizzi MP, Brunelli A, Marini U, Allevi G, Aguggini S, Tira A, et al. Accuracy of mammography and echography versus clinical palpation in the assessment of response to primary chemotherapy in breast cancer patients with operable disease. Breast Cancer Res Treat. 2001;69(2):143-51.

29. Luparia A, Mariscotti G, Durando M, Ciatto S, Bosco D, Campanino PP, Castellano I, Sapino A, Gandini G. Accuracy of tumour size assessment in the preoperative staging of breast cancer: comparison of digital mammography, tomosynthesis, ultrasound and MRI. Radiol Med. 2013; 118(7):1119-36.

30. Davis PL, Staiger MJ, Harris KB, Ganott MA, Klementaviciene J, KS MC Jr, Tobon $\mathrm{H}$. Breast cancer measurements with magnetic resonance imaging, ultrasonography, and mammography. Breast Cancer Res Treat. 1996;37(1):1-9.

31. Leddy R, Irshad A, Metcalfe A, Mabalam P, Abid A, Ackerman S, Lewis M. Comparative accuracy of preoperative tumor size assessment on mammography, sonography, and MRI: Is the accuracy affected by breast density or cancer subtype? J Clin Ultrasound. 2016;44(1):17-25.

\section{Submit your next manuscript to BioMed Central and we will help you at every step:}

- We accept pre-submission inquiries

- Our selector tool helps you to find the most relevant journal

- We provide round the clock customer support

- Convenient online submission

- Thorough peer review

- Inclusion in PubMed and all major indexing services

- Maximum visibility for your research

Submit your manuscript at www.biomedcentral.com/submit
) Biomed Central 